\title{
Meissner effect in a charged Bose gas with short-range repulsion
}

\author{
Shun-ichiro Koh* \\ Physics Division, Faculty of Education, Kochi University, Akebono-cho, 2-5-1, Kochi, 780, Japan
}

(Received 21 May 2003; published 2 October 2003)

\begin{abstract}
The question of whether the Bose-Einstein condensate (BEC) is a necessary condition of the Meissner effect is examined. The electromagnetic susceptibility of a charged Bose gas with short-range repulsion is studied using the perturbation theory with respect to the repulsive force. With decreasing temperature, the Bosestatistical coherence grows, and prior to the BEC phase the susceptibility shows a singularity implying the Meissner effect. This means that the BEC is a sufficient, but not a necessary, condition of the Meissner effect in the charged Bose gas with short-range repulsion.
\end{abstract}

DOI: 10.1103/PhysRevB.68.144502

PACS number(s): 67.20.+k, 74.25.Ha, 05.30.Jp

\section{INTRODUCTION}

The Meissner-Ochsenfeld effect (which from now on we shall call the Meissner effect) is known as a striking phenomenon showing directly the existence of the Bose-Einstein condensate (BEC). Actually, when cooling the metal in an applied magnetic field, just at the moment when the metal becomes superconducting, the metal begins to exclude the magnetic field.

A simple model exhibiting the Meissner effect is the charged Bose gas. Before the advent of the BCS model, Schafroth gave a clear definition of the Meissner effect using the charged Bose gas. ${ }^{1,2}$ The BCS model, starting from a realistic picture of the electrons in metal, established a realistic model exhibiting the Meissner effect. ${ }^{3}$

A common feature of these two models is an appearance of the off-diagonal long-range order (ODLRO) in low temperature such as ${ }^{4}$

$$
\left\langle\Phi(x)^{\dagger} \Phi(0)\right\rangle \Rightarrow f^{*}(x) f(0), \quad|x| \rightarrow \infty .
$$

To make the problem clearer, a model-independent derivation of the Meissner effect from fundamental principles and the ODLRO is desirable. Recently, such a derivation was attempted, with the result that the Meissner effect is derived from the gauge invariance and the ODLRO., ${ }^{5,6}$ This result means that the ODLRO is a sufficient condition of the Meissner effect.

For the converse statement that the ODLRO is a necessary condition of the Meissner effect, however, the problem is not so simple. Consider the following counterexample. In the two-dimensional systems, the order which one observes at zero temperature is not the ODLRO, but the off-diagonal finite-range order (ODFRO), which falls off exponentially at a long distance. ${ }^{7}$ But its dynamical response is not an ordinary one. Experimentally, the thin-film superconductors exhibit the Meissner effect. Theoretically, the two-dimensional charged Bose gas, although it does not show any mathematical singularity in the thermodynamic quantities, excludes the applied magnetic field at low temperature, which cannot be distinguished from the conventional Meissner effect in a practical sense. ${ }^{8}$ This example poses a question of whether the ODLRO is a necessary condition of the Meissner effect.
In other words, we face another question of whether we must have a more general definition of the Meissner effect than our present one.

In this paper, we assert that the ODLRO is not a necessary condition of the Meissner effect. The Meissner effect occurs even at temperatures in which the many-body wave function obeying Bose statistics grows to a large but not yet macroscopic size (ODFRO). ${ }^{9}$

An objection to this assertion is that one does not observe in the superconductors the Meissner effect at $T>T_{c}$. The phenomenon occurring in the superconducting metals at the vicinity of $T_{c}$ is, however, not a gradual growth of the coherent wave function from a finite to a macroscopic scale, but a formation of the Cooper pairs from two electrons. Once the Cooper pair forms a composite boson at low temperature and high density, the system does not change through the pre-BEC state, but it immediately jumps to the BEC phase. ${ }^{10}$ Hence the Meissner effect abruptly occurs at $T_{c}$ without any precursor. In this meaning, the absence of the Meissner effect at $T>T_{c}$ in the superconductors does not contradict the above assertion.

In principle, the charged Bose gas is a most elementary model of the Meissner effect. But, we do not have any experimental example except for the superconducting metal. This situation leads us to a preconceived idea that the ODLRO is a necessary and sufficient condition of the Meissner effect. Although the BEC is a most peculiar feature of Bose statistics, it does not automatically mean that all anomalous properties are attributed to the BEC. The bosons obey Bose statistics even when the Bose condensate is absent. Hence to what extent these anomalous phenomena imply the existence of the Bose condensate is, logically, a problem to be considered separately. From this standpoint, we can classify all phenomena in the dense cold bosons into two classes, the distinguishing factor being the necessity of the Bose condensate. The first is a class for which the Bose condensate is a necessary and sufficient condition, and the second is a class for which the Bose condensate is only a sufficient one, thus being possible even when the Bose condensate is absent.

In real systems, the existence of the interaction between particles complicates the above classification. An interplay between Bose statistics and the repulsive or attractive interaction determines the class of the phenomenon. In this paper, 
we study the Meissner effect using the charged Bose gas with short-range repulsion having the following Hamiltonian:

$$
\begin{aligned}
H= & \int d^{3} x\left(\frac{E^{2}+B^{2}}{2}+\left|\left(\partial_{\mu}+i e A_{\mu}\right) \Phi(x)\right|^{2}\right) \\
& +g \sum_{p, p^{\prime}} \sum_{q} \Phi_{p-q}^{\dagger} \Phi_{p^{\prime}+q}^{\dagger} \Phi_{p^{\prime}} \Phi_{p},
\end{aligned}
$$

where $\Phi$ is the spinless boson, and $g(>0)$ represents the short-range repulsive interaction due to the internal structure of the bosons $(\mu=x, y, z)$.

As will be explained in Sec. II A, the Meissner effect occurs when the transverse excitation of the system is suppressed compared with the longitudinal excitation. ${ }^{11}$ Following Feynman's argument, ${ }^{12}$ Sec. II B explains that this suppression is a fundamental feature of Bose statistics, being independent of the presence or absence of the BEC. In the charged ideal Bose gas $(g=0)$, where the long-range Coulomb force is assumed to be screened by the opposite charges in the medium, the suppression of the transverse excitation comes only from Bose statistics, and it exhibits the Meissner effect only in the BEC phase. In the charged Bose gas with short-range repulsion $(g>0)$, however, the collective excitation due to the repulsive interaction complicates the situation. As a general property of the gas, the longitudinal excitation prevails over the transverse one, thus destroying further the balance between the transverse and the longitudinal excitation. When this effect is added to the suppression due to Bose statistics, it is natural to ask whether the Meissner effect occurs before the many-body wave function grows to a macroscopic scale.

Section III studies the Meissner effect by calculating the electromagnetic susceptibility of the charged Bose gas with short-range repulsion using the perturbation expansion with respect to the repulsive interaction $g$. To examine a meaning of the BEC for the Meissner effect, we cannot assume the Bose condensate from the beginning by replacing the operator of zero-momentum particle with a $c$ number. ${ }^{13}$ Rather, it is crucially important to incorporate the Bose-statistical coherence of the many-body wave function without assuming the Bose condensate. Starting from the normal phase, the perturbation must be developed in such a way that as the order of the expansion increases, the susceptibility includes a new effect due to a larger coherent wave function. Section III shows an occurrence of the Meissner effect prior to the BEC phase in cooling the charged Bose gas with short-range repulsion. Section IV discusses the role of the repulsive interaction from a different point of view, and compares this Meissner effect with the conventional one in the superconductors.

\section{MEISSNER EFFECT IN THE CHARGED BOSE GAS}

\section{A. Divergence of the susceptibility}

We apply to the charged Bose gas a weak stationary magnetic field derived from a transverse vector potential $A_{\mu}(q, \omega) e^{i \omega t}$. The current density within the linear response is

$$
\left\langle J_{\mu}(q, \omega)\right\rangle=-\frac{e^{2}}{m^{2} c}\left[n m-\chi^{T}(q, \omega)\right] A_{\mu}(q, \omega),
$$

where $n$ and $m$ is a number density and a mass of the particle, respectively. The first term in the right-hand side of Eq. (3) is the usual diamagnetic current, and the second one is the paramagnetic term arising from a change in the system's wave function induced by $A_{\mu}(q, \omega)$. The second term $\chi^{T}(q, \omega)$ is a transverse part of the Fourier component of the current-current response tensor,

$$
\chi_{\mu \nu}\left(q, \omega_{n}\right)=\frac{1}{V} \int_{0}^{\beta} d \tau \exp \left(i \omega_{n} \tau\right)\left\langle T_{\tau} J_{\mu}(q, \tau) J_{\nu}(q, 0)\right\rangle,
$$

where

$$
J_{\mu}(q, \tau)=\sum_{p, n}\left(p+\frac{q}{2}\right)_{\mu} \Phi_{p}^{\dagger} \Phi_{p+q} e^{i \omega_{n} \tau}
$$

$(\hbar=1)$, and defined as

$$
\chi_{\mu \nu}(q, \omega)=\frac{q_{\mu} q_{\nu}}{q^{2}} \chi^{L}(q, \omega)+\left(\delta_{\mu \nu}-\frac{q_{\mu} q_{\nu}}{q^{2}}\right) \chi^{T}(q, \omega) .
$$

Inside of the matter, Eq. (3) is added to the Maxwell equation in vacuum,

$$
-\left(\frac{\omega^{2}}{c^{2}}-q^{2}\right) A_{\mu}^{e x}(q, \omega)=\frac{4 \pi}{c}\left\langle J_{\mu}^{(0)}(q, \omega)\right\rangle,
$$

as an additional source term. Hence one obtains a following relationship between $A_{\mu}(q, \omega)$ in matter and $A_{\mu}^{e x}(q, \omega)$ in vacuum:

$$
A_{\mu}(q, \omega)=\frac{A_{\mu}^{e x}(q, \omega)}{1-\frac{4 \pi e^{2}}{m^{2} c^{2}}\left(\frac{n m-\chi^{T}(q, \omega)}{\omega^{2} / c^{2}-q^{2}}\right)} .
$$

For the static limit, from gauge invariance, one knows $\mathrm{nm}$ $=\chi^{L}(q, 0)$ relating the longitudinal current-current response tensor $\chi^{L}(q, \omega)$ with number density $n$ and mass $m$ of the particle. ${ }^{14}$ Hence one gets

$$
A_{\mu}(q, 0)=\frac{A_{\mu}^{e x}(q, 0)}{1+\frac{4 \pi e^{2}}{m^{2} c^{2}}\left(\frac{\chi^{L}(q, 0)-\chi^{T}(q, 0)}{q^{2}}\right)} .
$$

Equation (9) means that, when the balance between the transverse and the longitudinal excitation is destroyed in the static and uniform limit $\left(\lim _{q \rightarrow 0}\left[\chi^{L}(q, 0)-\chi^{T}(q, 0)\right] \neq 0\right)$, the denominator in the right-hand side of Eq. (9) diverges as $q \rightarrow 0$, thus vanishing $A_{\mu}(q, 0)$ and $H=\nabla \times A$ at $q \rightarrow 0$ in matter (Schafroth's criterion of the Meissner effect).

From now on we express a term proportional to $q_{\mu} q_{\nu}$ in $\chi_{\mu \nu}$ by $\hat{\chi}_{\mu \nu}$ such as 


$$
\begin{aligned}
\chi_{\mu \nu}(q, \omega) & =\delta_{\mu \nu} \chi^{T}(q, \omega)+q_{\mu} q_{\nu}\left(\frac{\chi^{L}(q, \omega)-\chi^{T}(q, \omega)}{q^{2}}\right) \\
& \equiv \delta_{\mu \nu} \chi^{T}+\hat{\chi}_{\mu \nu} .
\end{aligned}
$$

Hence Eq. (9) is expressed as

$$
A_{\mu}(q, 0)=\frac{A_{\mu}^{e x}(q, 0)}{1+\frac{4 \pi e^{2}}{m^{2} c^{2}}\left(\frac{\hat{\chi}_{\mu \nu}(q, 0)}{q_{\mu} q_{\nu}}\right)} .
$$

(i) In the charged ideal Bose gas, $\hat{\chi}_{\mu \nu}$ is given by

$$
\hat{\chi}_{\mu \nu}(q, \omega)=-\frac{q_{\mu} q_{\nu}}{4} \sum_{p} \frac{f(\epsilon(p))-f(\epsilon(p+q))}{\omega+\epsilon(p)-\epsilon(p+q)},
$$

where $\epsilon(p)$ is the kinetic energy $p^{2} / 2 m$ of the spinless boson, and $f(\epsilon(p))$ is the Bose-Einstein distribution. In the BEC phase (chemical potential $\mu=0), f(\epsilon(p))$ in Eq. (12) is a macroscopic number for $p=0$ and nearly zero for $p \neq 0$. Thus, in the sum over $p$ in the right-hand side of Eq. (12), only two terms corresponding to $p=0$ and $p=-q$ remain, with a result that

$$
\hat{\chi}_{\mu \nu}(q, 0)=m n_{0} \frac{q_{\mu} q_{\nu}}{q^{2}},
$$

where $n_{0}$ is the number density of the $p=0$ Bose particles. In view of $q^{-2}$ in Eq. (13), the charged ideal Bose gas in the BEC phase exhibits the Meissner effect. In the normal phase $(\mu<0)$, however, when the sum over $p$ in Eq. (12) is carried out by replacing it with an integral, one notices that $q^{-2}$ dependence disappears in the result. Hence, in the charged ideal Bose gas, the BEC is a necessary and sufficient condition of the Meissner effect. In this case, the penetration depth $\lambda_{0}(T)$, defined in Eq. (3) by $J_{\mu}(q, 0)=-(c / 4 \pi)$ $\times\left[1 / \lambda_{0}^{2}(T)\right] A_{\mu}(q, 0) \quad$ at $\quad q \rightarrow 0, \quad$ is $\quad \lambda_{0}(T)=n_{0}(T)^{-0.5}$ $\times \sqrt{m c^{2} / 4 \pi e^{2}}$.

(ii) To analyze the Meissner effect in the charged Bose gas with short-range repulsion, instead of $\lim _{q \rightarrow 0}\left[\chi^{L}(q, 0)\right.$ $\left.-\chi^{T}(q, 0)\right] \neq 0$, we regard a divergence of the coefficient of $q_{\mu} q_{\nu}$ in $\chi_{\mu \nu}(q, 0)$ at $q \rightarrow 0$ [Eq. (10)], as a general definition of the Meissner effect.

\section{B. Effects of Bose statistics}

The physical explanation of $\lim _{q \rightarrow 0}\left[\chi^{L}(q, 0)-\chi^{T}(q, 0)\right]$ $\neq 0$ in the Bose gas dates back to the Feynman's argument on the scarcity of the excitation in the liquid helium $4 .{ }^{12} \mathrm{We}$ recapitulate his explanation of how Bose statistics affects the many-body wave function in configuration space.

(i) When the gas is in the BEC phase $(\mu=0)$, the wave function has the permutation symmetry on a macroscopic scale. Consider a collective transverse displacement moving particles on a solid straight line in Fig. 1(a) to that on a dotted wavy curve (black circles). At first, it seems a configuration change on a large scale. This displacement is, however, reproduced by a set of slight displacements (de- (a)

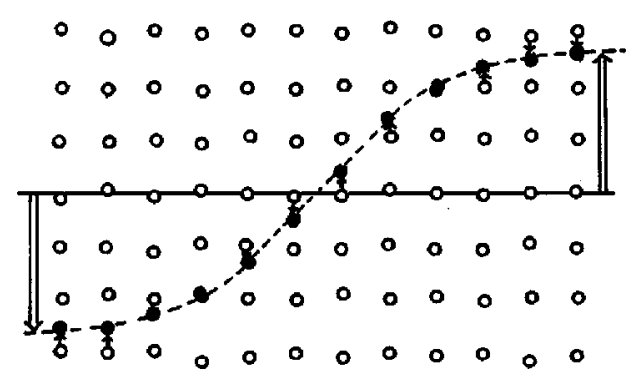

(b)
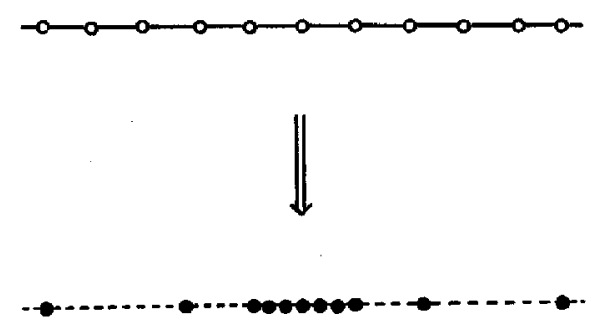

FIG. 1. A schematic picture of (a) transverse displacement, and (b) longitudinal displacement in coordinate space. White circles represent the bosons in the initial configuration.

picted by short arrows) moving the particle close to the wavy curve in the initial configuration to the exact positions on the curve. The transverse displacement like Fig. 1(a) changes only slightly the local density of particles (defined in some volume). Hence, at any part of the wavy curve, it is possible to find in the initial configuration a particle close to the wavy curve. In Bose statistics, due to permutation symmetry, one cannot distinguish between one particle on the wavy curve which comes from the position close to the wavy curve by the short arrow and another particle which comes from the straight line by the long arrow. Even if the displacement from the straight to the wavy line is a large displacement in the classical statistics, it is only a slight displacement in Bose statistics (short arrows). Hence the excited state lies in a small distance from the ground state in configuration space. Since the excited state is orthogonal to the ground state, the wave function corresponding to the excited state must spatially oscillate. Accordingly, the many-body wave function of the transversely excited state oscillates within a small distance in configuration space. Since the kinetic energy of the system is determined by the gradient of the wave function, this means that the energy of the transverse excitation is not small even at $q=0$, leading to the scarcity of the low-energy transverse-excitation density. This is a reason for $\chi^{T}(q, 0)$ $\rightarrow 0$ as $q \rightarrow 0$ at low temperature.

On the other hand, for the longitudinal displacement like Fig. 1(b), we see a different situation. Since the longitudinal displacement changes the local density so much, it is not possible to always find in the initial configuration a particle which is close to a given particle after displacement. (Even if it is possible for only a few particles after displacement, it is not possible for all particles.) Hence one cannot reproduce the configuration pattern after displacement only by permu- 
tation of the initial pattern [a lower pattern in Fig. 1(b) cannot be reproduced with an upper pattern]. This means that the longitudinally displaced configuration is far from the initial one in configuration space. Hence the wave function of the longitudinally excited state gradually oscillates in a long distance. Applying the definition of the kinetic energy to this case, one concludes that the low-energy longitudinal excitation is possible. This is a reason for $\chi^{L}(q, 0) \neq 0$ at $q \rightarrow 0$. The above explanation asserts that Bose statistics is an essential reason for the Meissner effect.

(ii) When the gas is at high temperature $(\mu \ll 0)$, the wave function has the permutation symmetry only in the limited area. Accordingly, in the transverse displacement like Fig. 1(a), one cannot regard the wave function after permutation as an equivalent of the initial wave function. The displacement which was regarded as a small displacement in the BEC phase becomes a large one in the normal phase. Since the difference between the transverse and the longitudinal excitation discussed in (i) vanishes, the Meissner effect disappears at high temperature.

(iii) From our viewpoint, a remarkable state of the Bose gas lies at the vicinity of the BEC transition temperature in the normal phase $(\mu \leqslant 0) .{ }^{15}$ The coherent many-body wave function grows to a large size but not yet to a macroscopic one. In such a situation, whereas the mechanism in (ii) works for the large displacement extending over two different wave functions, the mechanism in (i) works for the small displacement within a single wave function.

As shown in Eq. (13), the BEC is the necessary and sufficient condition of the Meissner effect for the charged ideal Bose gas. This conclusion is, however, applicable only to a simplified model like the charged ideal Bose gas. An inclusion of the repulsive interaction will change the situation, which will be studied in Sec. III.

\section{FORMALISM}

To examine the normal Bose gas at the vicinity of the BEC transition temperature, we cannot use the conventional method of assuming the Bose condensate by replacing the operator of zero-momentum particle with a $c$ number from the beginning. Consider the current-current response tensor of the charged Bose gas at the vicinity of the BEC transition temperature as illustrated in Fig. 2. The $\chi_{\mu \nu}$ in coordinate space includes the following form:

$$
\begin{aligned}
& \left\langle G\left|T_{\tau} J_{\mu}(x, \tau) J_{\nu}(0,0)\right| G\right\rangle \\
& =\frac{\left\langle 0\left|T_{\tau} \hat{J}_{\mu}(x, \tau) \hat{J}_{\nu}(0,0) \exp \left[-i \int_{0}^{1 / \beta} d \tau H_{I}(\tau)\right]\right| 0\right\rangle}{\left\langle 0\left|\exp \left[-i \int_{0}^{1 / \beta} d \tau H_{I}(\tau)\right]\right| 0\right\rangle},
\end{aligned}
$$

where $|G\rangle$ is a ground state of $\Sigma_{p} \epsilon(p) \Phi_{p}^{\dagger} \Phi_{p}$ $+g \Sigma_{p, p^{\prime}} \Sigma_{q} \Phi_{p-q}^{\dagger} \Phi_{p^{\prime}+q}^{\dagger} \Phi_{p^{\prime}} \Phi_{p}$. The excitation energy due to the long-range Coulomb force is too high to affect the dynamics of the bosons in the Meissner effect. ${ }^{16}$ Hence the (a)

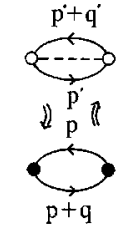

(c)

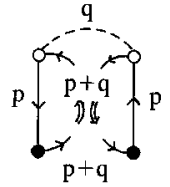

(b)

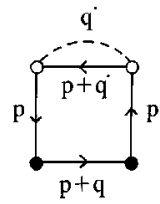

(d)

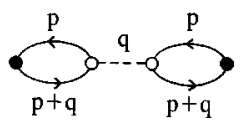

FIG. 2. The first-order Feynman diagram of the current-current response tensor among the one-particle excitations of the repulsive Bose gas in Eq. (14). (The solid and the dotted lines represent the boson and the repulsive interaction, respectively.) Particle exchange between the tensor and the medium by Bose symmetry is explained in four steps from (a) to (d).

photon-boson vertex appears only at an initial and a final vertex of the $\chi_{\mu \nu}$, and $H_{I}(\tau)$ in Eq. (14) represents $g \Sigma_{p, p^{\prime}} \Sigma_{q} \hat{\Phi}_{p-q}^{\dagger} \hat{\Phi}_{p^{\prime}+q}^{\dagger} \hat{\Phi}_{p^{\prime}} \hat{\Phi}_{p}$ (dotted lines in Fig. 2). In the ground state of Bose gas, due to the repulsive interaction $g$ contained in $\exp \left[-i \int H_{I}(\tau) d \tau\right]$ of Eq. (14), one-particle excitations frequently occur as illustrated by the upper bubble with a dotted line in Fig. 2(a). The current-current response tensor of such a medium is depicted by the lower bubble in Fig. 2(a). (The black and white small circles in Fig. 2 represent the vector and the scalar vertex, respectively.)

When $|G\rangle$ is the ground state at the vicinity of the BEC transition temperature in the normal phase, the perturbation must be developed in such a way that as the order of the expansion increases, the susceptibility gradually includes a new effect due to Bose statistics. An important feature of the response to the photon by the large coherent many-body wave function is that bosons appearing in the response tensor also participate in the coherent wave function of the medium. (The response tensor and the medium form a coherent wave function as a whole.) Hence we must seriously consider the influence of Bose statistics on the graph like Fig. 2(a). When one of the two bosons in the lower bubble and one of the two bosons in the upper bubble have a same momentum ( $p$ $=p^{\prime}$ ), and another boson in the lower and the upper bubbles have another same momentum $\left(p+q=p^{\prime}+q^{\prime}\right)$, a graph made by exchanging these two particles must be included in the expansion of $\chi_{\mu \nu}$. Such a graph is obtained in two steps as illustrated in Fig. 2. When two boson lines with $p$ and $p^{\prime}(=p)$ are exchanged in Fig. 2(a), a square appears in Fig. 2(b). Further, when two boson lines with $p+q$ and $p+q^{\prime}$ $(=p+q)$ are exchanged in Fig. 2(c), a graph linked by the repulsive interaction appears in Fig. 2(d). The resulting current-current correlation tensor has a form elongated by the density-density correlation.

A contribution of Fig. 2(d) to $\chi_{\mu \nu}$ is given by

$$
\begin{aligned}
\chi_{\mu \nu}^{(1)}(q, \omega)= & g \sum_{p}\left(p+\frac{q}{2}\right)_{\mu}\left(p+\frac{q}{2}\right)_{\nu} \\
& \times\left[-\frac{f(\epsilon(p))-f(\epsilon(p+q))}{\omega+\epsilon(p)-\epsilon(p+q)}\right]^{2} .
\end{aligned}
$$


With decreasing temperature, the coherent wave function grows to a large size, and the particle exchange due to Bose statistics like Fig. 2 occurs many times. Hence one cannot ignore the higher-order term $\chi_{\mu \nu}^{(n)}$ corresponding to the larger coherent wave function. Particularly important is the coherent wave function consisting of $p=0$ bosons. Correspondingly, a quantity of most interest which contribute to $\hat{\chi}_{\mu \nu}$ in
Eq. (10) is a term proportional to $q_{\mu} q_{\nu}$ in $\chi_{\mu \nu}^{(n)}(q, 0)$, the $p$ $=0$ component of which has the following form:

$$
\hat{\chi}_{\mu \nu}^{(n)}(q, 0)=\frac{q_{\mu} q_{\nu}}{4} g^{n} F_{\beta}(q)^{n+1},
$$

where

$$
F_{\beta}(q)=\exp (\beta \mu)\left(\frac{[1-\exp (\beta \mu)]^{-1}-\{\exp [\beta \epsilon(q)]-\exp (\beta \mu)\}^{-1}}{\epsilon(q)}\right)
$$

$F_{\beta}(q)$ is a positive monotonously decreasing function of $q^{2}$ which approaches zero as $q^{2} \rightarrow \infty$. As $\mu \rightarrow 0,\left|F_{\beta}(q)\right|$ increases at any $q$.

The general definition at the end of Sec. II A says that, when a power series in $g$,

$$
\hat{\chi}_{\mu \nu}(q, 0)=\frac{q_{\mu} q_{\nu}}{4} \sum_{n=0}^{\infty} g^{n} F_{\beta}(q)^{n+1} \equiv \frac{q_{\mu} q_{\nu}}{4} \sum_{n=0}^{\infty} a_{n} g^{n},
$$

diverges at $q \rightarrow 0$, the Meissner effect occurs. At high temperature in normal phase $(\beta \mu \ll 0)$, a small $F_{\beta}(q)$ guarantees the convergence of $\hat{\chi}_{\mu \nu}(q, 0)$ [Eq. (18)]. With decreasing temperature $(\mu \rightarrow 0)$, however, a gradual increase of $F_{\beta}(q)$ makes the higher-order term significant, finally leading to the divergence of $\hat{\chi}_{\mu \nu}(q, 0)$. A convergence radius $r_{c}$ of the power series $\sum_{n=1}^{\infty} a_{n} x^{n}$ is given by

$$
\frac{1}{r_{c}}=\lim _{n \rightarrow \infty}\left|a_{n}\right|^{1 / n}
$$

(Cauchy-Hadamard's theorem). Applying this theorem to Eq. (18) $\left[a_{n}=F_{\beta}(q)^{n+1}\right]$, one obtains $1 / r_{c}=F_{\beta}(q)$. Hence, as a convergence condition $0<g<r_{c}$, we have

$$
\left|g F_{\beta}(q)\right|<1 \text {. }
$$

Generally, for the Meissner effect to occur, the balance between the longitudinal and the transverse excitation must be destroyed on a macroscopic scale $(q \rightarrow 0)$. Together with the Bose-statistical coherence, the repulsive interaction enhances this tendency, finally violating the condition of Eq. (20).

An expansion form of $F_{\beta}(q)$ around $q^{2}=0$ is given by

$$
\begin{aligned}
F_{\beta}(q)= & \frac{\beta \exp (\beta \mu)}{[1-\exp (\beta \mu)]^{2}} \\
& -\frac{\beta^{2}}{2} \exp (\beta \mu) \frac{[1+\exp (\beta \mu)]}{[1-\exp (\beta \mu)]^{3}} \epsilon(q)+\cdots
\end{aligned}
$$

At high temperature, Eq. (20) is satisfied, and $\hat{\chi}_{\mu \nu}(q, 0)$ has the following form:

$$
\hat{\chi}_{\mu \nu}(q, 0)=\frac{q_{\mu} q_{\nu}}{4} \frac{F_{\beta}(q)}{1-g F_{\beta}(q)} .
$$

In cooling the gas, $\left|F_{\beta}(q)\right|$ increases monotonously, and the convergence condition is first violated at $q=0$ when $\left|g F_{\beta}(0)\right|=1$, that is, using Eq. (21),

$$
g \beta=4 \sinh ^{2}\left(\frac{\beta \mu}{2}\right) .
$$

From now, we call $T_{0}$ an onset temperature of the Meissner effect satisfying Eq. (23). In view of Eq. (23), we conclude that the charged Bose gas with short-range repulsion $(g$ $>0$ ) shows the Meissner effect prior to the BEC phase ( $\mu$ $<0$ ), thus leading to $T_{B E C}<T_{0}$. At $T=T_{0}$, substituting Eq. (21) in Eq. (22) with Eq. (23), we get

$$
\hat{\chi}_{\mu \nu}(q, 0)=\frac{m}{2 \sinh |\beta \mu|} \frac{q_{\mu} q_{\nu}}{q^{2}},
$$

which shows the Meissner effect in Eq. (11).

Compared with Eq. (13), the number density $n_{0}$ of $p=0$ boson which is a macroscopic number at $T_{B E C}$ is replaced by $0.5 / \sinh |\beta \mu|$ in Eq. (24). Accordingly, the penetration depth has a form such as $\lambda(T)=\sqrt{2 \sinh |\beta \mu|} \sqrt{m c^{2} / 4 \pi e^{2}}$. Since $\mu<0$ at $T_{0}, \lambda\left(T_{0}\right)$ is larger than $\lambda_{0}\left(T_{B E C}\right)$ in the ideal Bose gas, because at $T_{0}$ only $p=0$ boson whose number is large, but not yet macroscopic is responsible for this Meissner effect. Hence, at the onset temperature $T_{0}$, the magnetic field penetrates deeply into the gas. [It is in contrast with the abrupt exclusion of the magnetic field, that is, a very small $\lambda\left(T_{c}\right)$ in the superconductor.]

(i) In the weak-coupling case $(g \simeq 0)$, we can make a rough estimation of the condition of Eq. (23). Using $\mu_{0}(T)$ of the ideal Bose gas for $\mu(T)$ in Eq. (23), such as

$$
\beta \mu_{0}(T)=-\left(\frac{2.61}{2 \sqrt{\pi}}\right)^{2} \frac{T_{B E C}}{T}\left[\left(\frac{T}{T_{B E C}}\right)^{1.5}-1\right]^{2},
$$

we can determine $T_{0}$ as a solution of simultaneous equations (23) and (25). Figure 3 shows a phase diagram in which the onset temperature $T_{0}$ of the Meissner effect is plotted at a given strength of the repulsive interaction $g$. (Since only the relative strength of $g$ to the particle mass has a meaning, 


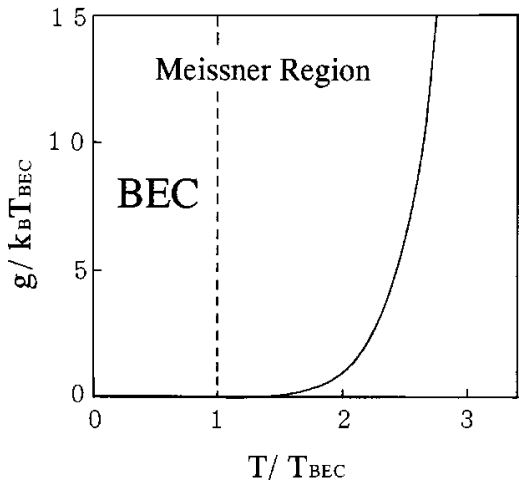

FIG. 3. A phase diagram showing the onset temperature of the Meissner effect $T_{0}$ at a given strength of the repulsive interaction $g$. Both quantities are normalized by $T_{B E C}$ and $k_{B} T_{B E C}$, respectively.

$g / k_{B} T_{B E C}$ is plotted.) Figure 3 confirms that for the charged ideal Bose gas $(g=0)$, the Meissner effect occurs just at $T$ $=T_{B E C}$, but it shows that the charged Bose gas with shortrange repulsion $(g \neq 0)$ begins to exclude the applied magnetic field prior to the BEC phase in cooling. (Although the repulsive interaction suppresses the $T_{B E C}$, it does not change the overall feature of Fig. 3.) The BEC is not a necessary condition of the Meissner effect for the charged Bose gas with short-range repulsion.

(ii) At high temperature $\left(T / T_{B E C} \gg 1\right)$, the repulsive interaction which is necessary for the Meissner effect to occur in Eq. (23) increases as $T \exp \left[a\left(T / T_{B E C}\right)^{2}\right]$. In view of the exponential factor, the Meissner effect does not occur at high temperature in real materials, thus not affecting the essential feature of the Meissner effect, a phenomenon in low temperature.

(iii) When cooling the system further below $T_{0}$ in Fig. 3 at a given $g$, the $\hat{\chi}_{\mu \nu}(q, 0)$ [Eq. (18)] diverges not only at $q$ $=0$, but also for a region $0<q \leqslant q_{c}(T)$, where $q_{c}(T)$ is determined by a condition $\left|g F_{\beta}\left(q_{c}(T)\right)\right|=1$ at a given $T$. For $q_{c}(T)<q$, the $\hat{\chi}_{\mu \nu}(q, 0)$ converges even below $T_{0}$. To obtain the overall feature of $\hat{\chi}_{\mu \nu}(q, 0)$ below $T_{0}$, we get a concrete form of $\hat{\chi}_{\mu \nu}$ by expanding $1-g F_{\beta}(q)$ in the righthand side of Eq. (22) with respect to $q^{2}-q_{c}(T)^{2}$. Hence one gets the following result:

$$
\begin{aligned}
& \hat{\chi}_{\mu \nu}(q, 0) \\
& =\left\{\begin{array}{l}
\infty, \quad 0 \leqslant q \leqslant q_{c}(T), \\
\frac{-q_{\mu} q_{\nu}}{4 g\left(\frac{\partial \ln F_{\beta}(q)}{\partial q^{2}}\right)_{q_{c}}\left[q^{2}-q_{c}(T)^{2}\right]},
\end{array} \quad q_{c}(T)<q .\right.
\end{aligned}
$$

Equation (26) in Eq. (11) means that the charged Bose gas with short-range repulsion excludes not only the uniform applied magnetic field $(q=0)$, but also the spatially modulated magnetic field $H(q)$ with $0<q \leqslant q_{c}(T)$.
With decreasing temperature, the number of particles with $p \neq 0$ which satisfies the divergence condition of $\hat{\chi}_{\mu \nu}(q, 0)$ gradually increases. Hence such particles participate in excluding the applied magnetic field, leading to a gradual decrease of the penetration depth.

The $q_{c}(T)$ reminds us of the coherence length $\xi$ in the superconductor, because $q_{c}(T)$ and $\xi^{-1}$ have a similar property: As they become larger, the Bose-statistical coherence grows. (For more comparison, see Sec. IV.) Here, we discuss the temperature dependence of $q_{c}(T)$.

(i) Just below $T_{0}, q_{c}(T)$ in Eq. (26) is small, and we can obtain the temperature-dependence of $q_{c}(T)$ as follows. We expand $F_{\beta}(q)$ in the condition of $\left|g F_{\beta}(q)\right|=1$ with a small $q_{c}(T)$ as in Eq. (21). Taking only the first and the second term in the right-hand side of Eq. (21), we get an approximate form of $q_{c}(T)$ as

$$
q_{c}(T)^{2}=4 m k_{B} T \tanh \frac{|\beta \mu(T)|}{2}\left[1-\frac{4 k_{B} T}{g} \sinh ^{2}\left(\frac{\beta \mu}{2}\right)\right] .
$$

At $T=T_{0}, q_{c}(T)$ is zero because of Eq. (23). At $T<T_{0}$, $q_{c}(T)$ is expanded with respect to $T_{0}-T$. In the case of weak repulsive interaction, using $\mu_{0}(T)$ [Eq. (25)] as $\mu(T)$, one gets

$$
q_{c}(T)=2 \sqrt{A m k_{B} T \tanh \frac{\left|\beta \mu_{0}(T)\right|}{2}} \sqrt{1-\frac{T}{T_{0}}},
$$

where

$$
A=1+1.08\left(\alpha^{2}-0.5 \alpha^{0.5}-0.5 \alpha^{-1}\right) \sqrt{\frac{4 k_{B} T_{0}}{g}+1},
$$

and $\alpha=T_{0} / T_{B E C}$.

(ii) When the decreasing temperature reaches $T=T_{B E C}$, $F_{\beta}(q)$ [Eq. (17)] diverges at all $q$, thus leading to a divergence of $\hat{\chi}_{\mu \nu}(q, 0)$ at all $q$. Hence $q_{c}(T)$ in Eq. (26) goes to $\infty$ in the BEC phase.

\section{DISCUSSION}

(i) In this paper, we explained the mechanism in which the repulsive interaction destroys the balance between the longitudinal and the transverse excitation on a macroscopic scale. This explanation can be viewed from a different point of view.

Generally, the influence of the repulsive interaction on the dynamical properties of the boson systems differs from that on the thermal properties. For the thermal properties, the repulsive interaction generally suppresses the growth of the Bose-statistical coherence. For example, it is known that due to the strong short-range repulsion, not all helium 4 atoms participate in the BEC even at zero temperature (depletion effect).

For the dynamical properties such as the superfluidity, however, the repulsive interaction generally enhances singular properties due to Bose statistics. For the ideal Bose gas, the particle with nonzero momentum behaves like a free par- 
ticle (except for the statistical constraint) to the external dynamical perturbation. For the repulsive Bose gas, however, the particles are likely to spread uniformly in coordinate space due to the repulsive force. This feature makes the particles with $p \neq 0$ behave similarly with other particles, especially with the particle having zero momentum. If it behaves differently from others, a resulting locally high density of particle raises the interaction energy. This is a reason why all particles seem to show singular dynamical behavior even at temperature in which not all particles participate in the BEC. (This fact corresponds to the well-known property that the one-particle energy spectrum changes from $p^{2} / 2 m$ to $s p$ due to the repulsive interaction, enhancing the stability of the superfluid flow to the thermal disturbance by raising the energy barrier around $p=0$.)

The Meissner effect is a dynamical response of the Bose gas to the external perturbation (the applied static magnetic field) as well. The boson excited from the $p=0$ to the $p$ $\neq 0$ state does not respond as a free particle to the magnetic field, but it excludes the magnetic field cooperatively with the $p=0$ bosons by the repulsive interaction. Hence, even when the Bose-statistical coherence grows to a large but not to a macroscopic scale (ODFRO), the charged Bose gas with short-range repulsion shows the Meissner effect. In this meaning, as well as the superfluidity, the Meissner effect is another example of the mechanism in which the repulsive interaction enhances the singular dynamical response of the boson system.

(ii) As discussed in Sec. I, the Meissner effect occurring in the charged Bose gas with short-range repulsion differs from that in the superconductors in some respects. Comparing two different Meissner effects is useful for a deeper understanding of them.

Let us replace the boson operator $\Phi_{p}$ in the right-hand side of Eq. (5) with the fermion operator $\Psi_{p, \sigma}$, and use such a $J_{\mu}(q, \tau)$ in Eq. (4). By factorizing the resulting $\left\langle T_{\tau} J_{\mu}(q, \tau) J_{\nu}(q, 0)\right\rangle$ into a product of the two anomalous fermion Green's functions, we get

$$
\begin{aligned}
\chi_{\mu \nu}(q, 0)= & \frac{1}{2} \int \frac{d^{3} p}{(2 \pi)^{3}}\left(p+\frac{q}{2}\right)_{\mu}\left(p+\frac{q}{2}\right)_{\nu} \\
& \times\left(1-\frac{\epsilon_{p} \epsilon_{p+q}+|\Delta|^{2}}{E_{p} E_{p+q}}\right) \frac{1}{E_{p}+E_{p+q}},
\end{aligned}
$$

where $\epsilon_{p}=p^{2} / 2 m-\mu, \Delta$ is the order parameter, and $E_{p}$ $=\sqrt{\epsilon_{p}^{2}+\Delta^{2}}$. Performing the integral over $p$ in Eq. (30) yields

$$
\chi^{T}(q, 0)=\left(n m-a|\Delta|^{2}\right)-b q^{2},
$$

*Email address: koh@cc.kochi-u.ac.jp

${ }^{1}$ M.R. Schafroth, Helv. Phys. Acta 24, 645 (1951).

${ }^{2}$ M.R. Schafroth, Phys. Rev. 100, 463 (1955).

${ }^{3}$ J. Bardeen, L.N. Cooper, and J.R. Schriefer, Phys. Rev. 108, 1175 (1957).

${ }^{4}$ O. Penrose, Philos. Mag. 42, 1373 (1951); O. Penrose and L. Onsager, Phys. Rev. 104, 576 (1956); C.N. Yang, Rev. Mod. Phys. 34, 694 (1962). where $n m=\chi^{L}(q, 0)$, and $a$ and $b$ is a parameter independent of $q^{2}$. Substituting Eq. (31) in the definition of Eq. (10), one obtains

$$
\hat{\chi}_{\mu \nu}(q, 0)=q_{\mu} q_{\nu}\left(\frac{a|\Delta|^{2}}{q^{2}}+b\right),
$$

which means that only below $T_{c}(|\Delta| \neq 0), \hat{\chi}_{\mu \nu}(q, 0)$ shows the mathematical singularity like $q^{-2}$, leading to the Meissner effect.

As well as the charged ideal Bose gas, the BCS model does not show the mathematical singularity in $\hat{\chi}_{\mu \nu}(q, 0)$ prior to the BEC, but after the BEC, only the first-order term in the perturbation expansion of $\chi_{\mu \nu}(q, 0)$ is enough to show the $q^{-2}$ singularity. In the BCS model, the particle interaction is assumed to be completely diagonalized by the formation of the Cooper pair, thus leaving no residual interaction between the pairs. This fact makes the next-order calculation of the susceptibility unnecessary. On the other hand, the charged Bose gas with short-range repulsion shows another type of singularity prior to the BEC in the normal phase, if the perturbation expansion of $\chi_{\mu \nu}(q, 0)$ is summed to infinite order.

In the superconductors, two different kinds of the coherence length are known. The first is $\xi_{0}$ appearing in the Pippard nonlocal electrodynamics, and the second is $\xi(T)$ appearing in the Ginzburg-Landau equation. They are not the same quantities, but both quantities represent the smallest size of the wave packets which the supercurrent carriers can form, thus reflecting an extent of transformation of individual particles from fermion to boson. Due to the composite-boson nature underlying the Cooper pairs, the type-II superconductors show the complicated nonuniform response to the uniform applied magnetic field.

On the other hand, the response of the charged Bose gas with short-range repulsion to the magnetic field is different, because it consists of the elementary boson. [ $\xi_{0}$ or $\xi(T)$ corresponds to the particle radius in this case.] The critical wave number $q_{c}(T)$ defined as Eq. (28) is an inverse of another type of the coherence length. The $1 / q_{c}(T)$ represents a characteristic length of the spatial variation of magnetic field. Generally, compared with the uniform field case, screening of the spatially modulated applied magnetic field needs the stronger Bose-statistical coherence in the gas. When the applied field varies with a length larger than the value of $1 / q_{c}(T)$, it is screened by the charged Bose gas with short-range repulsion. The increase of $q_{c}(T)$ in Eq. (28) means the growth of the Bose-statistical coherence in cooling the system.
${ }^{5}$ G.L. Sewell, J. Stat. Phys. 61, 415 (1990).

${ }^{6}$ H.T. Nieh, Gang Su, and Bao-Heng Zhao, Phys. Rev. B 51, 3760 (1995).

${ }^{7}$ T.M. Rice, Phys. Rev. 140A, 1889 (1965); P.C. Hohenberg, Phys. Rev. 158, 383 (1967).

${ }^{8}$ R.M. May, Phys. Rev. 115, 254 (1959).

${ }^{9}$ Note that ODFRO is a different concept from the diagonal longrange order (DLRO) found in various ordered state. 
${ }^{10}$ The fluctuation occurring at the vicinity of $T_{c}$ in the superconductors is smaller than that appearing in the liquid helium 4 close to $T_{B E C}$.

${ }^{11}$ As a review, see G. Baym, in Mathematical Methods in Solid State and Superfluid Theory, edited by R.C. Clark and G.H. Derrick (Oliver and Boyd, Edingburgh, 1969), p. 121.

${ }^{12}$ R.P. Feynman, Progress in Low Temperature Physics, edited by C.J. Gorter (North-Holland, Amsterdam, 1955), p. 17.

${ }^{13}$ It is well known that the charged Bogoliubov model, in which $\Phi_{p=0}$ is replaced by the $c$ number in the Hamiltonian of Eq. (2), shows the Meissner effect like the superconductors [Eq. (13)] at the first order of $g$. A theory without such a replacement is a purpose of this paper.

${ }^{14}$ See, for example, P. Nozières and D. Pines, The Theory of Quantum Liquids (Benjamin, New York, 1965), Vol. 1.

${ }^{15}$ S. Koh, Phys. Rev. B 64, 134529 (2001).

${ }^{16}$ The energy spectrum of the charged Bose gas with long-range Coulomb repulsion has been studied by many authors. See, e.g., S.K. Ma and C.W. Woo, Phys. Rev. 159, 165 (1967), and references therein. 